\title{
How Physicians Spend Their Work Time: an Ecological Momentary Assessment
}

\author{
Fabrizio Toscano, $M D^{1,2,3}$ (๑) Eloise O'Donnell, $M P H^{7}$, Joan E. Broderick, $P h D^{4}$, \\ Marcella May, $M A^{4}$, Pippa Tucker, $M A^{4}$, Mark A. Unruh, $P h D^{7}$, \\ Gabriele Messina, MD, $P h D^{2}$, and Lawrence P. Casalino, $M D, P h D^{l}$
}

'Department of Population Health Sciences, Weill Cornell Medicine, New York, NY, USA; ${ }^{2}$ Department of Molecular and Developmental Medicine, University of Siena, Siena, Italy; ${ }^{3}$ Department of Medicine, Albert Einstein College of Medicine and Montefiore Medical Center, Bronx, NY, USA;

${ }^{4}$ Center for Social \& Economic Research, University of Southern California, Los Angeles, CA, USA.

BACKGROUND: Little is known about how physicians spend their work time.

OBJECTIVE: To determine how physicians in outpatient care spend their time at work, using an innovative method: ecological momentary assessment (EMA).

DESIGN: Physician activity was measured via EMA, using a smartphone app.

PARTICIPANTS: Twenty-eight practices across 16 US states. Sixty-one physicians: general internal medicine, family medicine, non-interventional cardiology, orthopedics.

MAIN MEASURES: Proportions of time spent on 14 activities within 6 broad categories of work: direct patient care (including both face-to-face care and other patient carerelated activities), electronic health record (EHR) input, administration, teaching/supervising, personal time, and other.

KEY RESULTS: After excluding personal time, physicians spent $66.5 \%$ of their time on direct patient care $(23.6 \%$ multitasking with use of the EHR and $42.9 \%$ without the EHR), $20.7 \%$ on EHR input alone, $7.7 \%$ on administrative activities, and $5.0 \%$ on other activities $(0.6 \%$ using the EHR). In total, physicians spent $44.9 \%$ of their time on the EHR.

LIMITATIONS: Unable to measure time spent at home on the EHR or other work tasks; participating physicians were not a random sample of US physicians.

CONCLUSIONS: The efficiency of highly trained professionals spending only two-thirds of their time on direct patient care may be questioned. EHR use continues to account for a large proportion of physician time. Further attempts should be made to redesign both EHRs and physician work processes.

KEY WORDS: physicians time; electronic medical records

J Gen Intern Med 35(11):3166-72

DOI: $10.1007 / \mathrm{s} 11606-020-06087-4$

(c) Society of General Internal Medicine 2020

Electronic supplementary material The online version of this article (https://doi.org/10.1007/s11606-020-06087-4) contains supplementary material, which is available to authorized users.

Received August 17, 2019

Accepted July 29, 2020

Published online August 17, 2020

\section{INTRODUCTION}

Physician time is one of the most valuable resources in healthcare. The ways in which physicians spend their work time are likely to have a major impact on the quality and cost of care, on patients' experience of care, and on physician satisfaction, burnout, and professionalism. ${ }^{1-3}$ In recent years, concern about the amount of time physicians spend on electronic health records (EHRs) has heightened interest in the ways physicians spend their time, yet there is relatively little information about this important subject. ${ }^{2,4,5}$

We used an innovative method - ecological momentary assessment (EMA) - both to provide detailed estimates of physician time and to explore the feasibility of this approach for future large-scale study of physician time use. ${ }^{6}$

\section{METHODS}

\section{Ecological Momentary Assessment}

EMA is an innovative technique that has been increasingly applied in studies of healthcare, but has not been used to study physician time. ${ }^{6,7}$ In this study, EMA made it possible to capture physicians' self-reported activity in real time while avoiding bias associated with retrospective recall as well as changes that might be induced in physician behavior by the presence of an observer (in studies in which an observer records time use - see Appendix Section 2). When EMA protocols use random sampling to select assessment moments and when compliance with the protocol is high, as in this study, the resulting sampled points provide an unbiased estimate of time use during the sampled time period. ${ }^{6-8}$

We developed and pilot tested an Android smartphone app that beeped physicians at random intervals during the work day. Physicians selected a date when they would be seeing patients in the office (e.g., not a surgical day) and a time window during which they expected to be at work. When beeped, physicians clicked on the activity in which they were engaged at that moment. For each beep, physicians had an 8min window to respond, with a reminder beep given at $6 \mathrm{~min}$. 
Table 1 Physicians work activities

\begin{tabular}{|c|c|c|}
\hline Activity & Description & Category \\
\hline $\begin{array}{l}\text { Interacting with a patient and/or } \\
\text { family }\end{array}$ & Talking with a patient and/or family; examining the patient; performing a procedure & Patient Care \\
\hline Electronic Health Record input & $\begin{array}{l}\text { Dictating or entering progress notes into the medical record; coding diagnoses and billing } \\
\text { codes, signing paper or electronic records }\end{array}$ & EHR Input \\
\hline Reviewing/planning & $\begin{array}{l}\text { Reviewing patient medical records and/or test results; making plans for a patient; searching for } \\
\text { guidelines or research information relevant to a patient }\end{array}$ & Patient Care \\
\hline \multicolumn{3}{|c|}{ Communication about patients with: } \\
\hline $\begin{array}{l}\text { Patient and/or family } \\
\text { Staff at office, hospital, or } \\
\text { nursing home } \\
\text { Other healthcare providers } \\
\text { Insurance company, not prior } \\
\text { authorization }\end{array}$ & Communicating via phone, email, letter, secure portal, etc., with or about a patient & Patient Care \\
\hline Prior authorization & Obtaining prior authorization for medications, tests, procedures, devices, referrals, etc. & Admin Work \\
\hline Pharmacy & $\begin{array}{l}\text { Ordering or refilling prescriptions for patients. For time spent on prior authorization of } \\
\text { medications, please click the "prior authorization" button }\end{array}$ & Admin Work \\
\hline Workers' Compensation & Reviewing records; preparing reports & Admin Work \\
\hline Teaching/supervising & Teaching or supervising students, residents, or fellows & $\begin{array}{l}\text { Teaching/ } \\
\text { Supervising }\end{array}$ \\
\hline Administrative tasks/meetings & Meetings or other administrative tasks & Admin Work \\
\hline Personal time & $\begin{array}{l}\text { Meals, personal phone calls or emails, informal conversation with office staff not related to } \\
\text { patient care, etc. }\end{array}$ & Personal Time \\
\hline Other & Any activity not covered by the categories we have provided & Other \\
\hline
\end{tabular}

List of the 14 activities from which physicians could choose. The Description column shows the short explanation of each activity that physicians could see in the app. The Category column shows the category (of six categories) in which we placed the activity, when selected as an exclusive category. Physicians often checked more than one activity for any given beep, so we also created combinations of activities and assigned each combination to one of the six categories (See Appendix Table A1)

If the physician did not respond, the beep was recorded as unanswered (for more detail, see Appendix Sections 1 and 3).

\section{Activities and Combinations of Activities}

Based on review of the literature and on pilot testing with physicians, we developed a list of 14 activities (Table 1) in which physicians might engage while in the office.

Physicians commonly (32.1\% of beeps) reported multitasking - being engaged in two or more activities simultaneously — when beeped (Appendix Table A1). Therefore, in conducting our analysis, we expanded the list of activities to include combinations of activities (Appendix Table A1). This made it possible to determine both the time spent on individual activities (e.g., "interacting with a patient and/or family") and time spent on multitasking (e.g., when a physician selected both "interacting with a patient and/or family" and "EHR input"). We then grouped all activities and combinations of activities into six categories: Patient Care, EHR Input, Administrative Work, Teaching/Supervising, Personal Time, Other (Appendix Table A1).

It is important to note that we defined EHR Input as "Dictating or entering progress notes into the medical record; coding diagnoses and billing codes, signing paper or electronic records." We defined Reviewing/Planning as "Reviewing patient medical records and/or test results; making plans for a patient; searching for guidelines or research information relevant to a patient." When EHR Input was reported as a single activity, we categorized this as EHR Input and not as Patient Care. Entering progress notes can be considered an important part of patient care, but we chose not to include EHR input, when reported as a sole activity, in the Patient Care category because there has been a decade of physician complaints about the many clicks needed to satisfy EHR documentation requirements and about the copying and pasting of massive volumes of progress notes. ${ }^{3,9}$ In contrast, we categorized Reviewing/ Planning, even when reported as a single activity, as Patient Care because it is largely a voluntary, proactive physician activity, which in part may not even include EHR use. We placed EHR Input into the Patient Care category when it was combined with other activities such as Reviewing/Planning or Interacting with a patient and/or family + EHR input (Appendix Table A1).

\section{Sample}

Using the national CarePrecise Physician Database, we randomly sampled 435 physicians in three specialties: orthopedics, non-interventional cardiology, and general internal medicine (excluding hospitalists). Multiple contact attempts were made via mail and, when possible, phone or email.

Responding physicians were asked to complete a short online survey that determined their eligibility to participate and provided demographic information and information about their practice. Physicians were eligible to participate if they spent at least 20 hours per week providing patient care and if they owned an Android phone or were willing to be loaned one for participation (the app was only compatible with Android). Physicians who participated received a \$200 American Express Gift Card.

The response rate was low (8.3\%: of which 10 participating physicians from 10 practices completed the study), so we 
recruited additional physicians, including family physicians, from a convenience sample of practices. This yielded 53 additional physicians from 18 additional practices across the USA. Two physicians were excluded after participating because they reported being in the office/hospital for less than $50 \%$ of their beeps. The final sample included 61 physicians from 28 practices in 16 states (Appendix text and Appendix Fig. A1 provide details). Recruitment and participation in the study took place between January 2017 and May 2018.

\section{Analysis}

After excluding Personal Time, we created exhibits describing the percentages of work time physicians spent in each category (across all physicians, by specialty, and by practice ownership), as well as the most frequent subcategories, by dividing the number of beeps for which an activity in the category was reported by the total number of beeps. Statistically significant differences between groups were assessed via pairwise comparisons using chi-square tests or $t$ tests, as appropriate.

\section{RESULTS}

The sample included 61 physicians from 16 states and 28 practices: 29 primary care physicians (general internal or family medicine), 14 non-interventional cardiologists, and 18 orthopedists (Table 2). Physicians' mean age was 46.6 years; $61.0 \%$ were men; $67.2 \%$ worked in hospital-owned practices and $32.8 \%$ in independent practices. All physicians reported using EHRs. Physicians received on average 9.2 beeps per day [4-16] and responded to $94.1 \%$ (531) of the 564 beeps sent to them.

During the office day, physicians spent $10 \%$ of their time on personal activities (e.g., meals). We excluded this time - the 52 of 531 beeps for which physicians reported spending time on personal activities - from all analyses. As displayed in Fig. 1, after removing beeps in which physicians reported being engaged in Personal Time, physicians spent $66.5 \%$ of their time on Patient Care, $20.7 \%$ on EHR Input only while not directly engaged in patient care, $7.7 \%$ on Administrative Work, and 5\% on other activities (e.g., Teaching/Supervising, Other).

Of the $66.5 \%$ of time that physicians spent on Patient Care, $41.8 \%$ was spent exclusively interacting with patients (Fig. 2). An additional $24.5 \%$ was spent on interacting with patients plus EHR input or on reviewing/planning plus EHR input.

Physicians spent $44.9 \%$ of their total time (excluding Personal Time) inputting data into an EHR (Fig. 3). This included $20.7 \%$ of their time spent solely on EHR Input, $23.6 \%$ on multitasking involving both EHR input and patient care (e.g., EHR input while interacting with a patient), and $0.6 \%$ on EHR input combined with teaching/supervising activities. While $42.3 \%$ of EHR time was spent on EHR input as a single activity, $57.7 \%$ was spent in combination with other activities, nearly all of which (52.6\%) directly involved patient care (Fig. 3).

There was no statistically significant difference in time use across the three specialties (Fig. 1). However, time use differed by practice ownership (Fig. 4): physicians working in physician-owned practices spent $78.2 \%$ of their time on $\mathrm{Pa}$ tient Care, $14.8 \%$ on EHR Input, and $4.9 \%$ on Administrative Work, compared with $61.6 \%(p<0.001), 23.2 \%(p<0.001)$, and $8.9 \%(p<0.001)$, respectively, for physicians in hospitalowned practices. No statistically significant difference in time use was found across the three specialties (Fig. 1).

\section{DISCUSSION}

We found that physicians in diverse specialties spent most of their time on patient care-related activities $(66.5 \%)$, as opposed to EHR Input only or Administrative Work (28.4\% when summed together).

Multitasking was common. For example, of the $45.0 \%$ of their total work time that physicians spent on the EHR, $57.7 \%$ was spent multitasking (e.g., interacting with a patient during an office visit).

Table 2 Physician and practice characteristics

\begin{tabular}{lllll}
\hline \hline & Total $(\boldsymbol{n}=\mathbf{6 1})$ & Primary care $(\boldsymbol{n}=\mathbf{2 9})$ & Cardiology $(\boldsymbol{n}=\mathbf{1 4})$ & Orthopedics $(\boldsymbol{n}=\mathbf{1 8})$ \\
\hline Sex $(\%$ male) & $61 \%$ & $48 \% *$ & $53 \%$ & $78 \%$ \\
Age in years $(x \pm s d)$ & $46.6 \pm 11.9$ & $48.6 \pm 12.1$ & $45.5 \pm 12.0$ & $44.1 \pm 11.5$ \\
Practice size & $26.2 \%$ & $31.0 \%$ & $14.3 \%$ & $27.8 \%$ \\
1-15 physicians & $32.8 \%$ & $31.0 \%$ & $35.7 \%$ & $33.3 \%$ \\
16-100 physicians & $41.0 \%$ & $38.0 \%$ & $50.0 \%$ & $38.9 \%$ \\
>100 physicians & & & $71.4 \%$ & $50.0 \%$ \\
Practice ownership? & $67.2 \%$ & $75.8 \%$ & $28.6 \%$ & $50.0 \%$ \\
Hospital/hospital system & $32.8 \%$ & $24.2 \%$ & $5.7,1-12$ & $3.3,1-8$ \\
Independent physician & $5.6,1-15$ & $6.9,1-15^{*}$ & $100 \% *$ & $61.1 \%$ \\
EHR time use in years $(x$, range) & $85.2 \%$ & $93.2 \% *$ & $0 \%$ & $27.8 \%$ \\
Use scribe for EHR? & $9.8 \%$ & $3.4 \%$ & $0 \%$ & $0 \%$ \\
Not at all & $0 \%$ & $0 \%$ & $0 \%$ & $11.1 \%$ \\
Occasionally & $5.0 \%$ & $3.4 \%$ & & \\
Often & Almost always & & & \\
\hline
\end{tabular}

Analysis of responses to an online survey completed by 61 participating physicians at the time of enrollment in the study. EHR, electronic health record. In pairwise comparisons between groups, statistically significant differences $(p<0.05)$ were found only between physicians in the orthopedics group compared with physicians in the other two groups (noted with a "*” symbol) 


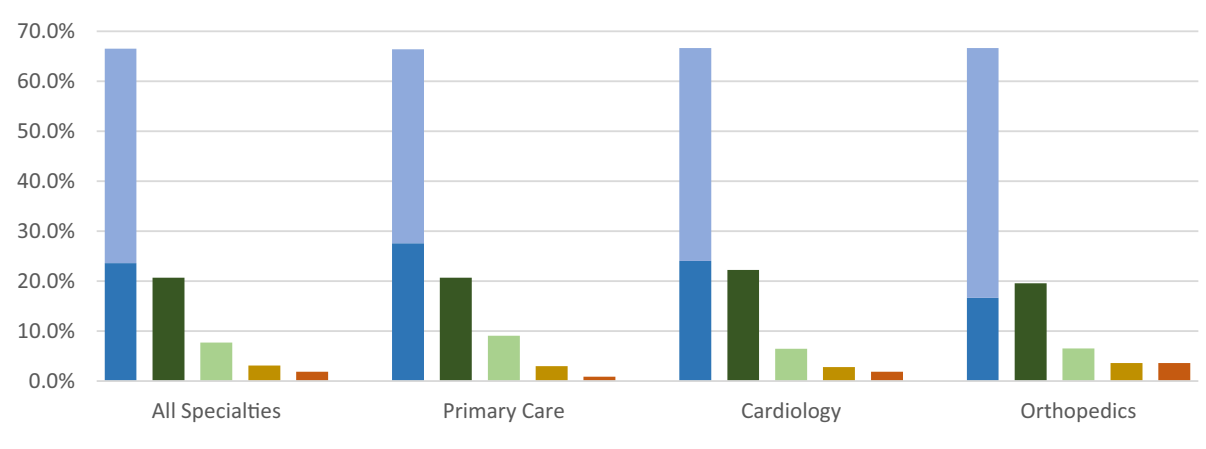

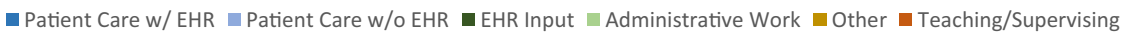

Fig. 1 Physician work time, by specialty. This exhibit excludes the $10.0 \%(53 / 531)$ of time physicians spent on personal activity (e.g., meals). Patient Care includes interacting with a patient, reviewing and planning patient care, communicating about patients with family, staff at office, hospital, nursing home, or other healthcare provider, or any combination of the above. EHR Input includes time spent inputting data into the electronic health record (EHR); this does not include EHR input when combined with patient care-related activities, which are included in Patient Care. Administrative Work includes administrative tasks/meetings, communication with insurance companies, pharmacies, workers' compensation, or obtaining prior authorization, or combinations of the above. Other includes any activity not covered by these categories. Teaching/Supervising includes teaching/supervising as a single activity or in combination with other activities; Appendix Table A1 shows how combinations of activities have been divided among these five categories (Patient Care is shown dived in with or without multitasking with the EHR input). Pairwise comparisons between groups per category of activity, using chi-squared tests, found no statistically significant differences

Our results differ somewhat from a recent time-and-motion study by Sinsky et al. ${ }^{9}$ In research involving 57 physicians (same specialties as our study) in 16 practices in 4 states, they found that only $27.0 \%$ of physician time was spent on "direct clinical face time" and $49.2 \%$ on "EHR and desk work." The differences may due to differing methodologies: our study recorded more multitasking, provided more activity options and combinations of activities (47 in total; see Appendix Section 3 and Appendix Table A1), and grouped these into different categories than Sinsky et al. In particular, we defined some EHR-related activities as patient care activities rather than as "EHR and desk work." For example, because coordinating care is a pivotal activity of ambulatory care, ${ }^{10}$ we categorized "EHR input plus reviewing/planning" as a patient care activity. In addition, Sinsky et al. asked observers to decide on the activity in which physicians were engaged, whereas we asked physicians to directly report this. It is also possible that the observers had an unknown effect on physician behavior while being observed.

To compare our findings more directly to Sinsky et al., we added together only activities or multitasking activities that involved direct contact with patients or with staff involved with patient care. These summed to $50.0 \%$ of physician time (data not shown) - substantially more than the $33.1 \%$ found by Sinsky et al., but less than the $66.5 \%$ of physician time we calculated by including activities like reviewing/planning as Patient Care time.

Using EHR access logs from a single community-based health system, Tai-Seale et al. reported that $49 \%$ of physicians' time is spent on "face-to-face visits" and $51 \%$ on "desktop medicine," including 34\% on progress notes and 9\% documenting telephone encounters and other activities. ${ }^{11}$ This study did not measure time spent when physicians were not logged into the EHR and could not always discern the activities in which physicians were engaged when they were logged in, nor did it measure multitasking.

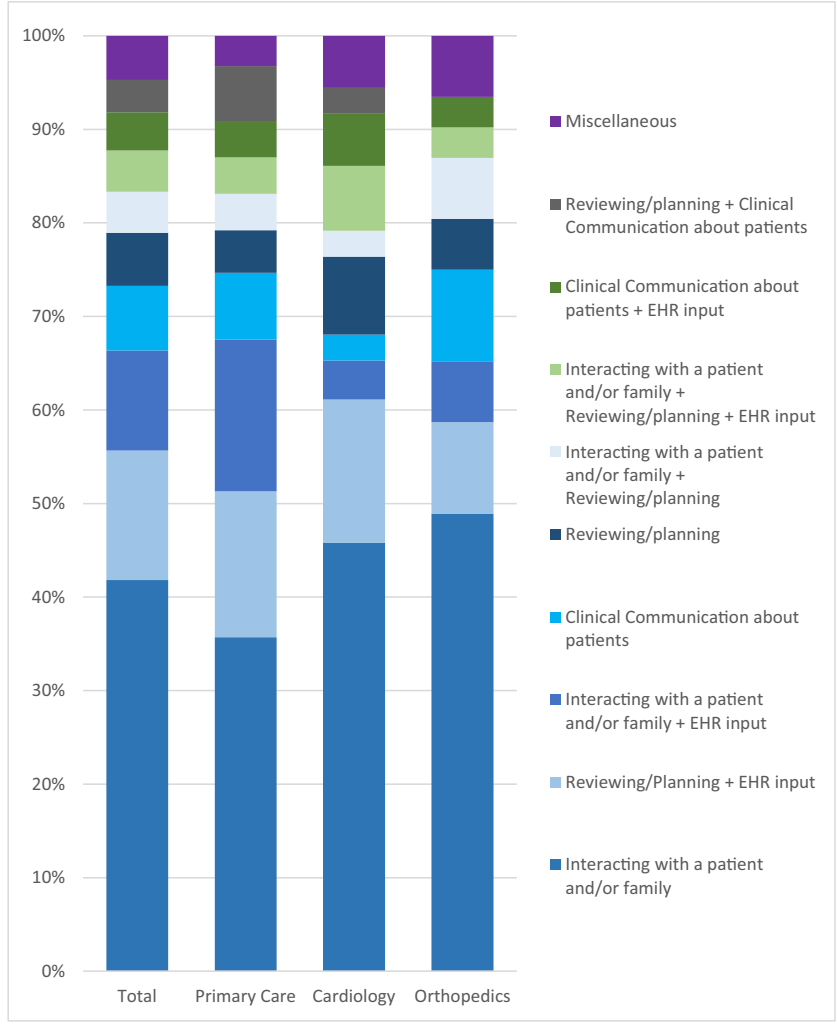

Fig. 2 Activities reported for the $66.5 \%$ of beeps categorized as Patient Care. This exhibit excludes the $10.0 \%(53 / 531)$ of time physicians spent on personal activity (e.g., meals), and shows the breakdown of the 318 of $478(66.5 \%)$ physician responses to beeps that indicated the physician was involved in Patient Care when beeped. EHR, electronic health record. Clinical Communication about patients includes communicating about patients with family, staff at office, hospital, nursing home, or other healthcare provider. For clarity, only the most frequent nine activities/activity combinations are displayed; those remaining are included in Miscellaneous. Due to the small numbers in some categories, no statistical tests were conducted between combinations of activities 


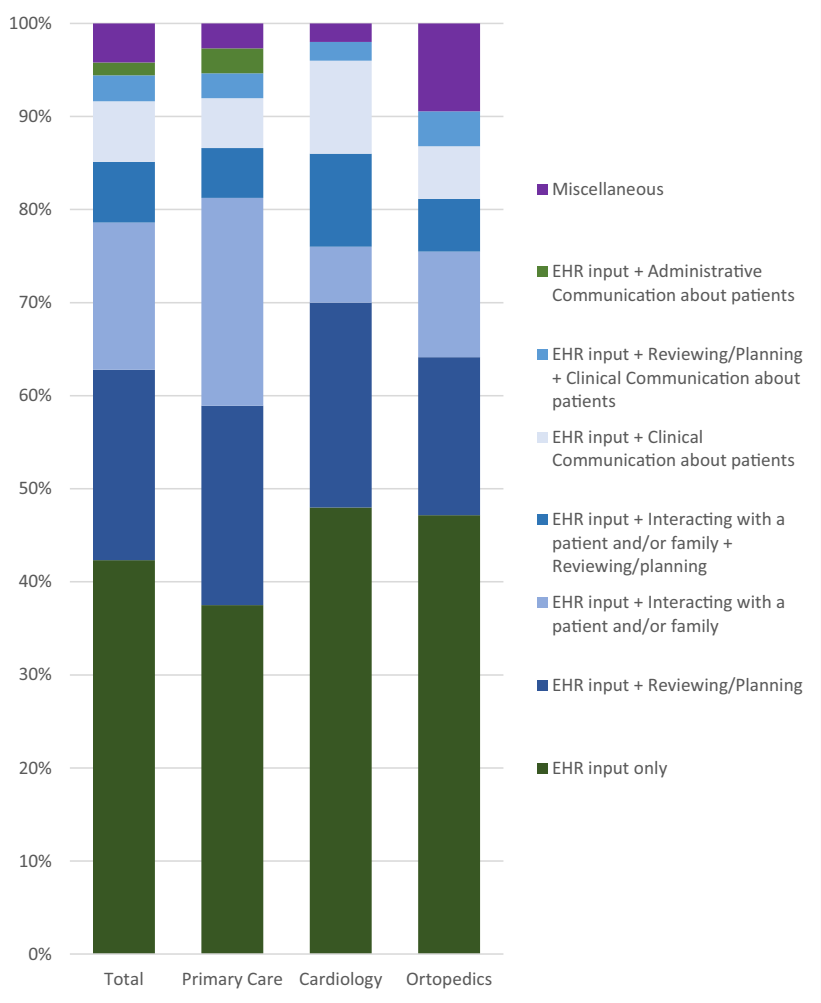

Fig. 3 Activities reported for the $45.0 \%$ of beeps for which EHR use was indicated. This exhibit excludes the $10.0 \%(53 / 531)$ of time physicians spent on personal activity (e.g., meals), and analyzes the 215 of $478(45.0 \%)$ of physician responses to beeps that include electronic health record (EHR) input. The activity "EHR input" was selected 215 times by physicians, 91 times as a single activity, and 124 times in combination with other activities. Clinical Communication about patients includes communicating about patients with family, staff at office, hospital, nursing home, or other healthcare provider. Administrative Communication about patients includes communication with insurance companies, pharmacies, workers' compensation, or obtaining prior authorization. For clarity, only the first seven activities/activity combinations are displayed in detail; those remaining are included in Miscellaneous. Due to the small numbers in some categories, no statistical tests were conducted between combinations of activities

Physician dissatisfaction with EHRs may result not only from the amount of time spent but also from other factors, e.g., dealing with multiple alerts, the necessity of making a large number of clicks per day, ${ }^{12,}{ }^{13}$ and lack of eye contact with patients while dealing with the EHR. ${ }^{14}$ Several strategies have been suggested to reduce EHR burden, such as the use of scribes, but it is not yet clear whether they increase net costs for physicians. ${ }^{15-17}$ In our sample, orthopedists used more scribes than primary care physicians and cardiologists, a difference that was statistically significant. Though there was no statistically significant difference in time use across specialties, primary care physicians did appear to spend more time on EHR Input plus Patient Care with EHR Input (48\%) than orthopedists (35\%). This is consistent with the perception than the documentation burden for primary care physicians (for example, documenting for performance measurement by payers) is high.
Given that hospitals have been rapidly acquiring physician practices, it may be important that physicians in hospitalowned practices spent significantly less time on patient carerelated activities than physicians in independent practices (61.6\% vs. $78.2 \%)$ and more time on EHR input (23.2\% vs. $14.8 \%){ }^{18,19}$ The reasons for this are not clear; it is possible that hospital-owned practices are more bureaucratic in what they expect physicians to enter in the EHR, but this is merely speculative.

This is the first study to use EMA to measure physician time use. EMA has several advantages for empirical observation of physician time use. Compared with time-and-motion studies that use observers to shadow physicians, EMA studies could be much less expensive to conduct on a larger scale, avoid bias that may occur when an observer is present with a physician and patient, and allow for the measurement of physician activities during the $5.5 \%$ of physician time that Sinsky et al. found "closed to observation." In contrast to EHR log studies, EMA is able to measure activities during times when physicians are not logged in to an EHR, and EMA can measure multitasking, which our results indicate is a very common physician activity.

Despite these strengths, our study had several limitations. First, the number of participating physicians was relatively small, the response rate was low, and, as a convenience sample, the data is not nationally representative. However, the study did include a broad range of practices - considerably broader than previous research. ${ }^{9}, 11,20,21$ Second, because physicians did not want to be beeped at home, we were unable to measure time that physicians spent on the EHR at home, which is likely substantial. ${ }^{5,11,22}$ An optimal study might combine EMA to measure physician time use at the office with EHR log measurements such as those in the study by Tai-Seale et al. to measure physician time use at home. ${ }^{11}$

We encountered two major challenges. Physician recruitment was difficult: informal recruitment feedback suggested that physicians were concerned about being beeped while face-to-face with patients, though participating physicians responded to $94.1 \%$ of the beeps they received, and those that did participate did not indicate that this was overly intrusive or time-consuming.

In contrast to the general US population, we found that the vast majority of contacted physicians use iPhones, not Android phones. ${ }^{23}$ Though we provided loaner Android phones upon request, the added effort this required from physicians almost certainly reduced willingness to participate. Creating an iPhone EMA app would not be technically difficult, though time and budget constraints precluded it for this study.

The cost of conducting an EMA study of physician time use within a practice, however large, or across multiple practices, could be reasonably low, if practice leaders strongly support the study. Practice leaders who want to understand patterns of physician time use within their organization, and changes over time, could encourage their physicians to participate. This could be done in the context of discussions with a practice's 


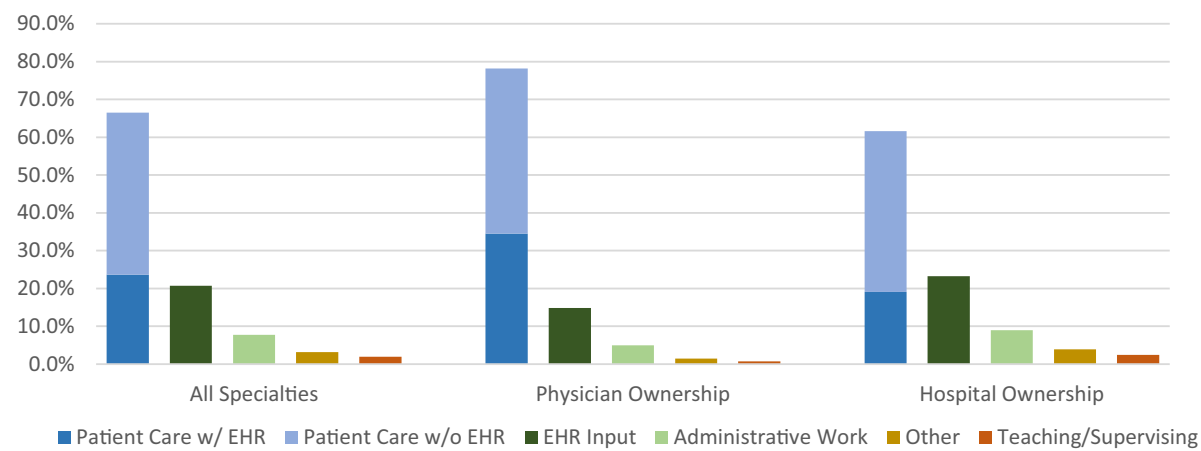

Fig. 4 Physician work time, by practice ownership. This exhibit excludes the $10.0 \%(53 / 531)$ of time physicians spent on personal activity (e.g., meals). Patient Care includes interacting with a patient, reviewing and planning patient care, communicating about patients with family, staff at office, hospital, nursing home, or other healthcare provider, or any combination of the above. EHR Input includes time spent inputting data into the electronic health record (EHR); this does not include EHR input when combined with patient care-related activities, which are included in Patient Care. Administrative Work includes administrative tasks/meetings, communication with insurance companies, pharmacies, workers' compensation, or obtaining prior authorization, or combinations of the above. Other includes any activity not covered by the categories we

have provided. Teaching/Supervising includes teaching/supervising as a single activity or in combination with other activities. Appendix Table A1 shows how combinations of activities have been divided among these five categories (Patient Care is shown dived in with or without multitasking with the EHR input). Pairwise comparisons between groups per category of activity, using chi-squared tests, showed statistically significant differences between physician-owned and hospital-owned practices in physician time allocation: $78.2 \% \mathrm{vs} .61 .6 \%(p<0.001)$ on Patient Care, $14.8 \%$ vs. $23.2 \%(p<0.001)$ on EHR Input, and $4.9 \%$ vs. $8.9 \%(p<0.001)$ on Administrative Work

physicians about how they use their time and about how they believe they could best use their time. A large-scale research study involving many practices would provide increased generalizability of results and very large studies would be inexpensive compared with time-and motion studies, since it would not be necessary to pay an observer for each day of physician participation. We note that physicians responded to $94.1 \%$ of the beeps they received, suggesting that participating was not burdensome for physicians. In addition, developing a version of the app for iPhones would likely increase physician participation and reduce research staff time necessary to assist participants.

\section{CONCLUSIONS}

In terms of physician time spent on patient care activities compared with EHR time, our results are somewhat more optimistic than prior studies. However, our finding that physician spend $45.0 \%$ of their time on an EHR alone or in combination with other activities is still very substantial, and suggests that intensified efforts should be made to reduce this time. We are not aware of studies that address how physicians should effectively allocate their work time. However, EHRs have been widely described as an irritant for physicians and as a major cause of physician burnout. $^{2-4,15,24}$ Therefore, reducing EHR time and/or ameliorating the user experience should be a primary goal. ${ }^{25}$

The fact that physicians - highly-trained professionals whose time is expensive - spend only $66.5 \%$ of their time on patient care suggests that a back-to-the drawing board approach to time-use by these highly trained professionals might be useful. This would likely involve changes in EHRs, changes in the way that physicians are paid, and changes in the processes that physicians and practice staff use to provide care, with payment changes making process changes economically viable. ${ }^{26}$

Acknowledgments: The authors would like to thank Tania Gutsche, Bas Weerman, and Swaroop Samek for their previous work in the developing of the app.

Corresponding Author: Fabrizio Toscano, MD; Department of Medicine, Albert Einstein College of Medicine and Montefiore Medical Center, Bronx, NY, USA (e-mail: ftoscanomd@gmail.com).

Funding Information This research was supported by a grant from The Physicians Foundation.

\section{Compliance with Ethical Standards:}

Conflict of Interest: Dr. Casalino reports personal fees from American Medical Association Advisory Committee on Physician Professional Satisfaction and Practice Sustainability, outside the submitted work. All other authors have no conflict of interest to disclosure.

\section{REFERENCES}

1. Dugdale DC, Epstein R, Pantilat SZ. Time and the patient-physician relationship. J Gen Intern Med. 1999;14 Suppl 1:S34-40.

2. Downing NL, Bates DW, Longhurst CA. Physician Burnout in the Electronic Health Record Era: Are We Ignoring the Real Cause? Ann Intern Med. 2018;169(1):50-51. https://doi.org/10.7326/M18-0139

3. Friedberg Mw. Factors Affecting Physician Professional Satisfaction and Their Implications for Patient Care, Health Systems, and Health Policy. Santa Monica: Rand Health, American Medical Association; 2013.

4. Collier R. Electronic health records contributing to physician burnout. CMAJ Can Med Assoc J J Assoc Medicale Can. 2017;189(45):E1405E1406. https://doi.org/10.1503/cmaj.109-5522

5. Arndt BG, Beasley JW, Watkinson MD, et al. Tethered to the EHR: Primary Care Physician Workload Assessment Using EHR Event Log Data and Time-Motion Observations. Ann Fam Med. 2017;15(5):419-426. https://doi.org/10.1370/afm.2121

6. Stone AA, Shiffman S. Ecological Momentary Assessment (Ema) in Behavioral Medicine. Ann Behav Med. 1994;16(3):199-202. https://doi. org/10.1093/abm/16.3.199 
7. Shiffman S, Stone AA, Hufford MR. Ecological momentary assessment. Annu Rev Clin Psychol. 2008;4:1-32.

8. Broderick JE. Electronic Diaries: Appraisal and Current Status. Pharm Med. 2008;22(2):69-74.

9. Sinsky C, Colligan L, Li L, et al. Allocation of Physician Time in Ambulatory Practice: A Time and Motion Study in 4 Specialties. Ann Intern Med. 2016;165(11):753-760. https://doi.org/10.7326/M16-0961

10. Press MJ. Instant Replay - A Quarterback's View of Care Coordination. $N$ Engl J Med. 2014;371(6):489-491. https://doi.org/10.1056/ NEJMp1406033

11. Tai-Seale $\mathbf{M}$, Olson $\mathbf{C W}, \mathbf{L i} \mathbf{J}$, et al. Electronic Health Record Logs Indicate That Physicians Split Time Evenly Between Seeing Patients And Desktop Medicine. Health Aff Proj Hope. 2017;36(4):655-662. https://doi. org/10.1377/hlthaff.2016.0811

12. Shanafelt TD, Dyrbye LN, Sinsky C, et al. Relationship Between Clerical Burden and Characteristics of the Electronic Environment With Physician Burnout and Professional Satisfaction. Mayo Clin Proc. 2016;91(7):836-848. https://doi.org/10.1016/j.mayocp.2016.05.007

13. Hingle S. Electronic Health Records: An Unfulfilled Promise and a Call to Action. Ann Intern Med. 2016;165(11):818-819. https://doi.org/10. 7326/M16-1757

14. Erickson SM, Rockwern B, Koltov M, McLean RM. Medical Practice and Quality Committee of the American College of Physicians. Putting Patients First by Reducing Administrative Tasks in Health Care: A Position Paper of the American College of Physicians. Ann Intern Med. 2017;166(9):659-661. https://doi.org/10.7326/M16-2697

15. Babbott S, Manwell LB, Brown R, et al. Electronic medical records and physician stress in primary care: results from the MEMO Study. $J$ Am Med Inform Assoc JAMIA. 2014;21(e1):e100-106. https://doi.org/10. 1136/amiajnl-2013-001875

16. Tutty MA, Carlasare LE, Lloyd S, Sinsky CA. The complex case of EHRs: examining the factors impacting the EHR user experience. $J \mathrm{Am}$ Med Inform Assoc JAMIA. April 2019. https://doi.org/10.1093/jamia/ ocz021

17. Casalino LP, Gans D, Weber R, et al. US Physician Practices Spend More Than \$15.4 Billion Annually To Report Quality Measures. Health Aff
(Millwood). 2016;35(3):401-406. https://doi.org/10.1377/hlthaff.2015. 1258

18. Burns LR, Goldsmith JC, Sen A. Horizontal and Vertical Integration of Physicians: A Tale of Two Tails. In: Goes J, Savage GT, Friedman L, eds. Advances in Health Care Management. Vol 15. Emerald Group Publishing Limited; 2013:39-117. https://doi.org/10.1108/S1474-8231(2013) 0000015009

19. Bishop TF, Shortell SM, Ramsay PP, Copeland KR, Casalino LP. Trends in hospital ownership of physician practices and the effect on processes to improve quality. Am J Manag Care. 2016;22(3):172-176.

20. Baron RJ. What's Keeping Us So Busy in Primary Care? A Snapshot from One Practice. N Engl J Med. 2010;362(17):1632-1636. https://doi.org/ 10.1056/NEJMon0910793

21. Young RA, Burge SK, Kumar KA, Wilson JM, Ortiz DF. A Time-Motion Study of Primary Care Physicians' Work in the Electronic Health Record Era. Fam Med. 2018;50(2):91-99. https://doi.org/10.22454/FamMed. 2018.184803

22. Farber J, Siu A, Bloom P. How Much Time Do Physicians Spend Providing Care Outside of Office Visits? Ann Intern Med. 2007;147(10):693. https://doi.org/10.7326/0003-4819-147-10200711200-00005

23. Ventola CL. Mobile devices and apps for health care professionals: uses and benefits. P T Peer-Rev J Formul Manag. 2014;39(5):356-364.

24. Meigs SL, Solomon M. Electronic Health Record Use a Bitter Pill for Many Physicians. Perspect Health Inf Manag. 2016;13:1d.

25. Asan O, Smith PD, Montague E. More screen time, less face time implications for EHR design. J Eval Clin Pract. 2014;20(6):896-901. https://doi.org/10.1111/jep.12182

26. Casalino LP. A Martian's Prescription For Primary Care: Overhaul The Physician's Workday. Health Aff (Millwood). 2010;29(5):785-790. https:// doi.org/10.1377/hlthaff.2010.0133

Publisher's Note: Springer Nature remains neutral with regard to jurisdictional claims in published maps and institutional affiliations. 